\title{
Corela
}

Cognition, représentation, langage

HS-19 | 2016

Le point de vue pris au mot

\section{Sens et topoi lexicaux en corpus : le lieu de l'apartheid}

Hugues de Constantin de Chanay

\section{(2) OpenEdition}

\section{Journals}

\section{Édition électronique}

URL : http://journals.openedition.org/corela/4351

DOI : $10.4000 /$ corela.4351

ISSN : 1638-573X

\section{Éditeur}

Cercle linguistique du Centre et de I'Ouest - CerLICO

\section{Référence électronique}

Hugues de Constantin de Chanay, «Sens et topoi lexicaux en corpus : le lieu de l'apartheid », Corela [En ligne], HS-19 | 2016, mis en ligne le 08 juin 2016, consulté le 30 avril 2019. URL : http:// journals.openedition.org/corela/4351 ; DOI : 10.4000/corela.4351

Ce document a été généré automatiquement le 30 avril 2019.

\section{(c) (i) (2)(2)}

Corela - cognition, représentation, langage est mis à disposition selon les termes de la licence Creative Commons Attribution - Pas d'Utilisation Commerciale - Partage dans les Mêmes Conditions 4.0 International. 


\title{
Sens et topoi lexicaux en corpus : le lieu de l'apartheid
}

\author{
Hugues de Constantin de Chanay
}

\section{Introduction}

1 En 1993 nous écrivions un article intitulé « Sens lexical et argumentation : des CNS aux topoi ». Son ambition était de montrer qu'étaient compatibles - mieux: complémentaires - dans une même description du plan sémantique du lexique d'une langue deux démarches au contraire régulièrement opposées, l'une issue de l'analyse componentielle (mais surtout de l'acclimatation structuraliste qu'en a faite l'analyse différentielle), référentialiste dans ses versions non structurales d'origine ainsi que dans certaines versions structurales (Kerbrat-Orecchioni 1977), l'autre inspirée des théories d'Anscombre et Ducrot (1983), « versant argumentatif » qui a presque toujours été opposé à la sémantique dénotative. Bruxelles et Constantin de Chanay (1998) vont aussi dans le sens d'un lien entre les deux types de théorie. Dès l'origine information et argumentation ont été opposées (en dépit des conceptions rhétoriques traditionnelles telles qu'on les retrouve par exemple dans la théorie de lieux produite par Perelman et Olbrechts-Tyteca (1988: 112-132), ce n'est pas le choix de l'information qui argumente). C'est ainsi que Raccah présentait toujours sa position en 2011 : «Dans mon usage de ce cadre théorique [l'argumentation dans la langue], le concept d'orientation argumentative tient une place aussi importante dans la description sémantique des phrases, que celui d'information » (2011: 182).

$2 \mathrm{Au}$ contraire les théories sémantiques dénotatives du premier courant subordonnent l'argumentation à l'information et interprètent les traits sémantiques structuraux qu'elles dégagent comme des "CNS» (conditions nécessaires et suffisantes) pour la dénomination (Kleiber 1990), c'est-à-dire un ensemble de propriétés que l'on peut vérifier que possèdent les choses, ou référents, et dont la liste fonde la dénotation (le reste est donc connotation). C'étaient donc des modèles opposés que nous nous efforcions d'accorder. Le présent ouvrage offre l'occasion de revenir une vingtaine d'années plus 
tard sur ces propositions à la lumière de ces évolutions survenues entretemps que sont d'une part le développement de la sémantique interprétative de Rastier, qui a priori délaisse le lexique de la langue pour se tourner vers les discours et les corpus (surtout écrits), et d'autre part la Sémantique des Points de Vue développée par Raccah, qui s'intéresse au moins autant au discours qu'à la langue, et toujours dans la même perspective : moins pour son pouvoir (à nos yeux réel) de représentation que pour son aptitude (non moins réelle) à contraindre des enchaînements.

corpus nous sera fourni par l'actualité du mois de janvier 2015 et les controverses auxquelles a donné lieu l'usage, par Manuel Valls, il est vrai dans un syntagme qui le détermine et dont la forme complète est « un apartheid social, territorial, ethnique $»^{1}$, du mot apartheid pour désigner une situation française.

Dans un premier temps nous présenterons brièvement le corpus, car nous saisissons le mot apartheid en discours (le corpus est donc un corpus de discours obervés). Ensuite nous nous intéresserons au signe apartheid, notamment à son signifiant, mais aussi à son statut (nom propre). Nous passerons ensuite à la face signifiée, précisément à la valeur, en convoquant les autres signes du même paradigme (intervention de François Hollande) ou d'autres discours (notamment, les images accompagnant sur deux badges le mot apartheid figurant dans des slogans en anglais). Enfin, conformément à une vision possible de la doxa dans la lignée d'une remarque de Rastier, nous regarderons plus attentivement ce qui se passe à un niveau polysémiotique, et notamment dans les deux pictogrammes figurant sur des badges militants. Tout cela nous permettra enfin de présenter quelques hypothèses sur la nature et de rôle des topoi dans le sens lexical et pour l'argumentation.

\section{Corpus}

Le 20 janvier 2015 Manuel Valls a traditionnellement présenté ses vœux à la presse. Cette allocution en grande partie protocolaire est cependant - en dépit de son caractère qui pourrait être purement épidictique - l'occasion d'un discours non convenu, et ainsi attendu et très scruté (celui-ci a entraîné immédiatement de nombreuses réactions). Voici une transcription d'un extrait vidéo ${ }^{2}$ :

(1) MV : oui ces derniers jours/ ont souligné beaucoup/ (.) des maux/ (mots/) (.) qui rongent notre pays $\backslash$ (.) ou des défis/ (.) que nous avons/ (.) à relever $\backslash$ (.) et à cela/ il faut ajouter:/ (.) toutes les fractures/ (2.0) les tensions/ (.) qui couvent/ (.) depuis trop longtemps $\backslash$ (.) et dont on parle/ uniquement/ par intermittences/ (.) et après/ on on oublie (.) c'est ainsi (.) les émeutes de deux-mille-cinq / (.) qui aujourd'hui s'en rappelle et pourtan:t / (.) les stigmates/ sont toujours présentes $\backslash$ (.) la relégation péri-urbaine/ (.) les ghettos/ (.) ce que j'évoquais / (.) EN deux mille cinq/ déjà $\backslash$ (.) un apartheid ${ }^{3}$ (.) territorial/ (.) social/ (.) ethnique/ (.) qui s'est/ euh (.) imposé/ euh (.) à notre pays $\backslash$ (.) la misère sociale/ (.) auxquels s'additionnent / (.) les discriminations/ quotidiennes/ (.) parce que l'on (n') a pas le bon nom de famille/ (.) la BONne couleur de peau/ (.) ou bien parce que l'on est une femme $\backslash$ (.) i n's'agit/(.) en AUCUN cas/ (.) et vous m'connaissez $\backslash$ (.) de chercher/ (.) la MOINdre/ excuse/ (.) mais il faut AUSSI $\backslash$ ( .) regarder (.) la réalité (.) de notre pays $\backslash$ (.) et puis/ (.) il y a aussi/ (.) cette peur collective/ (.) face/ (.) au chômage/ de masse/ (.) au chômage/ de longue durée/ (.) au chômage/ des jeunes/ (2.2) face à la vie/ trop chère/ (.) au risque/ de déclin/ (.) lui-même entretenu/

Les chutes intonatives sont rares, en outre elles ne sont pas très marquées. En revanche les montées intonatives sont fréquentes et marquées, y compris voire surtout quand elles n'ont pas de rôle syntaxique attendu (par exemple pour indiquer qu'un syntagme va 
recevoir incessamment un complément comme dans " ces derniers jours/ ont souligné » ou qu'il est inachevé et va être poursuivi comme dans «vie/ trop chère»). Ce qui leur confère un rôle marqué c'est que souvent elles précèdent un silence: il y a dès lors immédiatement une tension entre leur fonction d'appel (Morel et Danon-Boileau 1998) qui fait attendre un complément.

7 Cette allocution forme système avec une autre ${ }^{4}$ : conférence adressée à la presse par François Hollande le 5 février 2015, deux semaines après les vœux de Manuel Valls et le tollé suscité par le mot apartheid. Cette conférence a été confrontée par la presse à l'intervention de Manuel Valls dans la mesure où elle aborde le même sujet (les difficultés rencontrées par la population de certains quartiers pauvres) mais pas avec les mêmes mots. En voici une transcription :

(2) FH : je dis que tout citoyen de la république/ (.) a les mêmes droits $\backslash$ (.) et a les mêmes devoirs $\backslash(2.0)$ je dis/ aussi:/ que: (.) la république/ reconnaît tous ses enfants/ (.) où qu'ils soient nés/ (.) où qu'ils vivent/ (.) quels que soient leur(s) parcours/ leur(s) couleur(s)/ (.) leur(s) religion(s) \(.) leur(s) conviction(s) $\backslash$ (.) et que la France/ a un devoir/ (.) ce n'est pas une dette/ le devoir $\backslash$ (.) c'est un devoir qui est lié : à la promesse/-même de la République $\backslash$ (.) le devoir/ de faire/ que : (.) chacun de ses enfants/ (.) chacun de ses citoyens/ (.) puisse réussir sa vie $\backslash$ (.) et ne pas avoir/ euh (.) le sentiment qu'il est ségrégé/ (.) séparé/ (.) discriminé/ [[⿷匚⿳⿰口口犬阝] $]$ (.) écarté/ (.) mis de côté/ (2.0) parce qu'il vivait dans le même/ (.) ensemble/ (.) dans

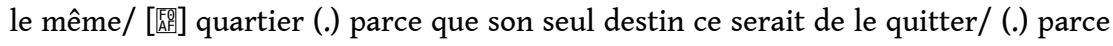
qu'il n'y aurait plu:s/ (.) possibilité/ (.) d'être véritablement ensemble\ (.) la GRANDE question qui nous est posée mais elle ne date pas d'aujourd'hui/ elle ne date pas [MD] du mois dernier/ (.) c'est est-ce que nous avons la capacité/ (.) de vivre/ ensemble/ (.) pas de vivre à côté les uns des autres/ (.) de vivre ensemble\

Ce qui saute aux yeux, le même sujet étant abordé à deux semaines, c'est, d'une part, que sur ce point la conférence de François Hollande est une réponse aux vœux de Manuel Valls ou du moins forme système avec eux. Et, d'autre part, que le « mot qui fâche », celui d'apartheid, n'est pas prononcé. On peut même parler d'évitement.

9 Nous essaierons d'atteindre un système qui n'est pas donné mais que le linguiste doit, s'il le peut, s'efforcer de reconstituer. Il doit en ce qui concerne le signifié, conformément à une terminologie de racine saussurienne et suivie par Ducrot, s'efforcer de reconstituer les systèmes des significations qui permettent les sens en discours. Comme le dit Rastier, «la doxa n'informe pas seulement la littérature et les autres discours: c'est un phénomène polysémiotique et l'on retrouve des topoï analogues dans les textes, les images, etc. » $(2011: 123)$.

Le corpus de discours que nous faisons entrer en ligne de compte comporte ainsi en outre deux occurrences du mot apartheid figurant sur des pictogrammes montés en badges militants, que voici ${ }^{5}$ : 


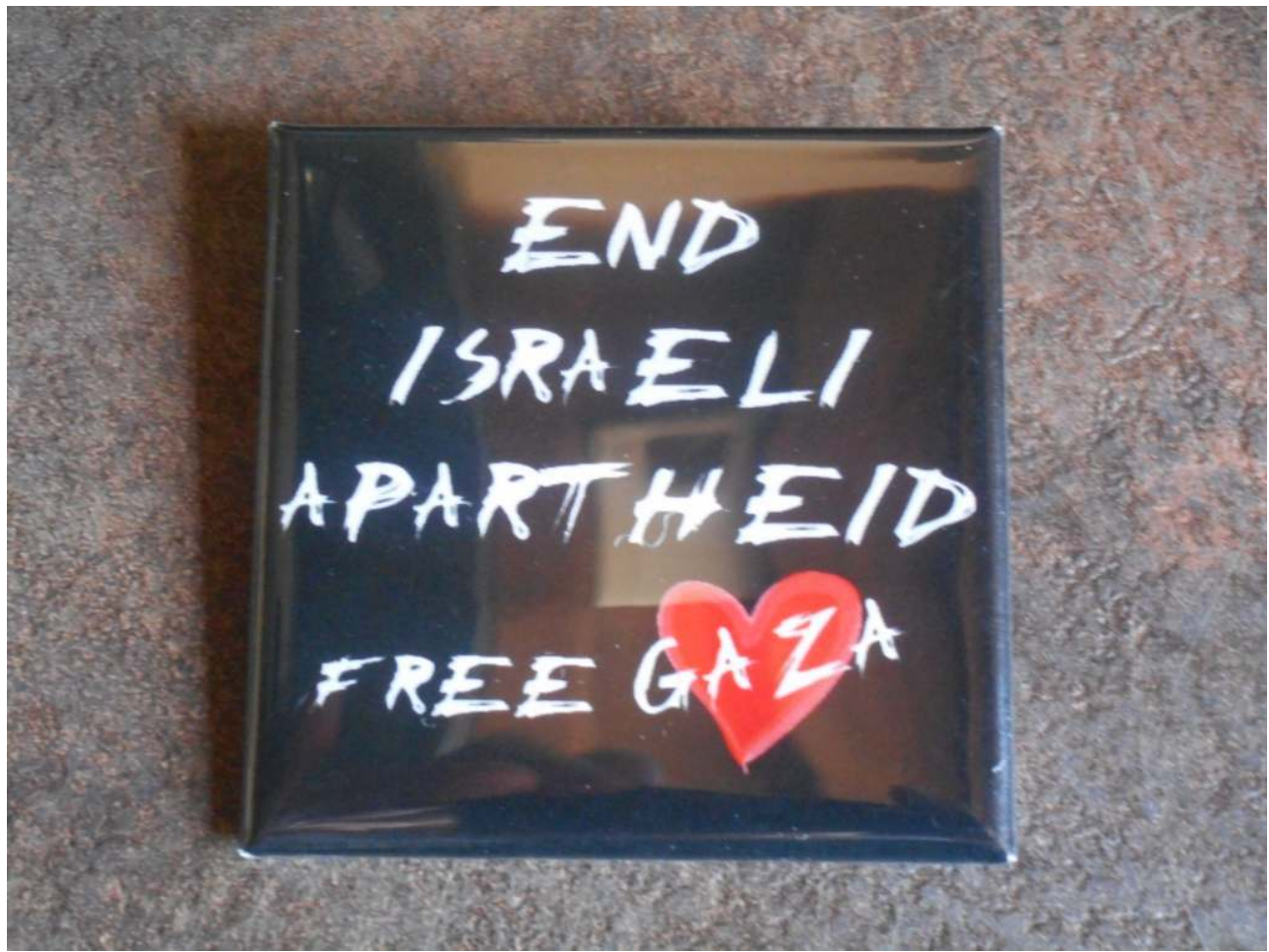

badge $n^{\circ} 1$

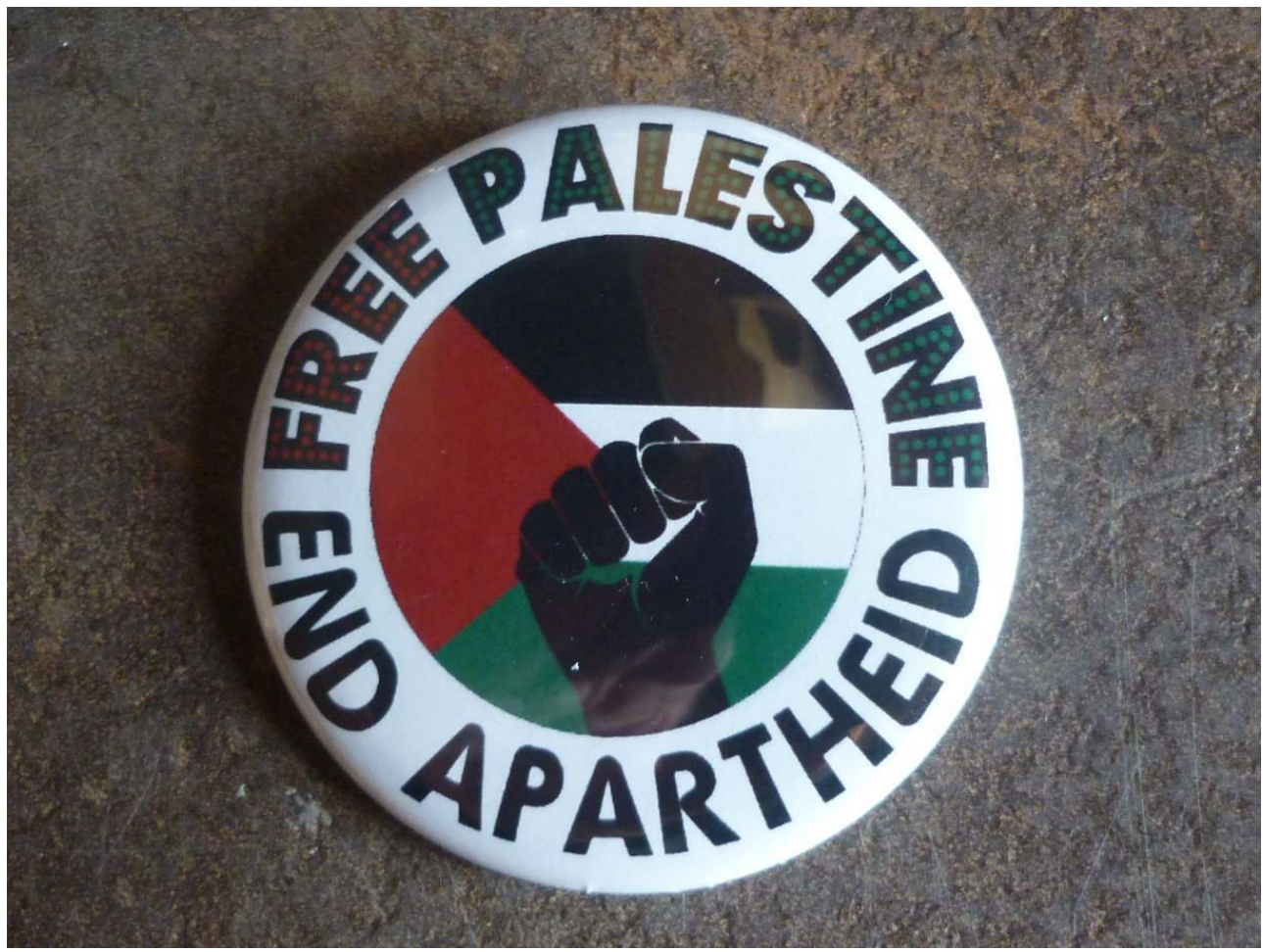

badge $n^{\circ} 2$

11 Ces images sont en fait, non des images d'« apartheid» (toujours contestables quand explicitement le mot apartheid ne figure pas dans l'énoncé iconique, ce qui est la plupart du temps le cas), mais des images associées à des slogans comportant le mot apartheid à l'intérieur d'un slogan linguistique en anglais et dans les deux cas appliqué au conflit israélo-palestinien, c'est-à-dire (point commun avec le discours de Manuel Valls) à une 
autre situation que cette situation historique de séparation entre blancs et noirs en Afrique du Sud à laquelle fait pour tout un chacun référence originellement le mot apartheid. Ainsi, si rien ne garantit sinon une forme d'intuition ici inévitable qu'il s'agisse bien des mêmes topoï dans les deux discours de Manuel Valls et de François Hollande et dans les deux pictogrammes, au moins il s'agit bien des mêmes mots (nous commencerons par là) et l'on peut supposer que pour chaque badge les topoï sont homogènes entre l'image et l'énoncé linguistique comportant le mot apartheid.

\section{Cadre de l'étude}

La sémantique interprétative se méfie de longue date de la notion de langue et surtout de la notion de mot. Rastier montre que le privilège du niveau lexical n'a pas de légitimité linguistique mais est lié à des considérations ontologiques: le découpage du monde en " choses » nécessaire au commerce comme aux architectures web ne s'impose nullement aux sciences du langage mais permet leur listage potentiel en labels stabilisateurs, l'ensemble des deux (choses et mots) formant une nomenclature.

Or c'est la "valeur » sémantique (au sens de Saussure 1972 [1917]) qui est fondatrice du signifié, non les choses auxquelles on réfère en employant les mots dans des discours. Le célèbre exemple de la confrontation de la paire anglaise mutton (viande de mouton pour l'assiette) / sheep (animal sur pied), qu'on dira en intersection définitionnelle, avec l'unique mot français mouton (viande ou animal) qui correspond aux deux, illustre la double aporie à laquelle on est très rapidement conduit avec la théorie de la valeur quand on espère qu'elle va permettre de pouvoir se concentrer sur le niveau sémantique de la langue défini comme isolable du monde. Au niveau macro, c'est l'aporie du territoire de signification commun que partagent sheep et mouton ou mutton et mouton; ou, pour en rester au seul français, brebis, mouton, agneau, bélier. Au niveau micro se pose par ailleurs le problème de la substance du contenu. Par exemple, qu'est-ce qui permet de dire que / mutton/ et $/$ sheep $/{ }^{6}$ s'opposent par les traits [vivant] vs [non vivant] et que /mouton/ les neutralise? Rien si on ne considère pas les possibilités de désignation en discours, l'occurrence de «mouton » dans des conversations de table pour désigner le contenu du plat, et outre-Manche, de « mutton » et jamais de « sheep ».

Insistons pour en venir à l'idée de champ lexical : la signification n'est pas déterminée par une relation établie entre mot et objet décrit (ou défini). Et c'est elle qui, au contraire et en vertu de sa position dans un champ, détermine la relation possible du mot à des objets. Quant à cette signification, elle est déterminée par opposition à la signification d'autres mots du même territoire sémantique ${ }^{7}$ tels que, pour mouton, bœufs, vaches, taureaux, porcs, poulets, etc. (divers bestiaux d'élevage), d'une part, ou merguez $z^{8}$, poisson, poulet, quenelles, etc. (partie noble ${ }^{9}$ du plat de résistance), d'autre part (et en opposition auxquels mouton peut voisiner avec flageolets, ragoût ou pommes de terre alors que dans le premier cas il le fera plutôt avec prairie, bergère, grillage ou laine). C'est dire que la signification est première à la fois pour l'appartenance à une même catégorie référentielle (bestiaux ou partie animale du plat de résistance) et pour le choix des partenaires lexicaux en discours (courir, brouter, bêler, etc., ou cuire, carré, navarin, napper, servir, assiette, piquer, mâcher, etc.).

L'appartenance à une même catégorie ontologique légitime l'héritage par tous les membres des traits de l'archisémème qui les subsume. Les taxèmes exemplaires cités par Rastier, par exemple celui des substantifs désignant des transports en commun (bus, 
métro, car, tramway), sont aussi de cet ordre. C'est à nos yeux un problème théorique. Ces taxèmes ne se rencontrent pas souvent en corpus (par définition : ce sont des paradigmes dont les éléments s'excluent) et peuvent difficilement être établis expérimentalement. Évaluer leur plausibilité communicationnelle est à la fois nécessaire et difficile: un taxème, dit Rastier à la suite de Pottier, loin d'être un ensemble exhaustif de mots comme on peut en réunir à de fins didactiques, est le " petit ensemble de termes réellement disponibles et vraisemblablement utilisables chez le locuteur dans une circonstance donnée de communication » $(1987: 33)$.

La théorie de l'argumentation dans la langue est à l'opposé de cette sémantique si on l'envisage comme une théorie du signe, mais elle ne l'est pas dès qu'on l'envisage comme une théorie reliée aux sens en discours.

L'objet empirique de Ducrot est l'énoncé - c'est-à-dire que c'est un objet discursif de rang supérieur à celui du signe. Et par opposition aux sémantiques primordialement référentielles, la théorie de l'argumentation remet au centre de la signification, hors connotation, des questions essentiellement non dénotatives : par l'emploi de ce mot en discours, comment le locuteur peut-il enchaîner? Que peut-il prouver, ou du moins défendre? En d'autres termes, qu'est-ce que l'emploi de ce mot permet de dire que ne permettrait pas un autre emploi? «Le signe complet est la phrase ", dit Ducrot (1993: 234). En effet l'unité d'observation pertinente pour le plan argumentatif est l'enchaînement argument-conclusion, lequel canoniquement ne se réalise pas dans le signe mais à un rang bien supérieur, dans une structure propositionnelle. Ce n'est pas pour autant récuser le plan de la langue : Ducrot pratique une distinction entre phrases et énoncés, les énoncés actualisant de phrases de langue; ou, pour le prendre à rebours et épouser l'attitude de l'interprète face à un énoncé, une phrase de langue est reconstituable sous l'énoncé qu'on interprète. L'idée de langue est liée au couplage énoncé-argumentation, et donc plus au rang de la phrase qu'à celui du signe. Le signe linguistique, souvent le mot mais plus précisément le morphème, n'est pas pour autant non pertinent : simplement, il appartient à ce signe plus complet. Sans autonomie propre, il en est une segmentation. Ce qui est pertinent pour la phrase et l'énoncé, l'enchaînement conclusif, le reste pour lui. Ce qui est significatif pour tel mot c'est son aptitude à figurer dans les énoncés qui argumentent en faveur de telle ou telle conclusion.

18 Si cette segmentation rencontre inévitablement de mots qu'on peut mettre en relation avec des objets en déterminant leur signification en un sens, ce n'est pas le cas général parce que le critère essentiel reste l'insertion possible dans une structure argumentconclusion. Contrastent emblématiquement des mots comme peu et un peu qui ont du point de vue de la référence les mêmes conditions de vérité (ils peuvent être employés à propos de la même quantité, la référence n'explique donc pas leur contraste) mais qui entrent dans des argumentations opposées :

(3) Il y a peu d'argent, n'en dépensons pas

(4) Il y a un peu d'argent, on peut le dépenser

19 C'est ainsi également que des « mots du discours » comme mais ou au contraire peuvent se décrire en dépit du fait qu'on ne peut pas les relier à des classes référentielles. Ces mots dépourvus comme mais de conditions de référence ou que l'on doit comme peu et un peu employer dans des argumentations opposées en dépit de leur synonymie référentielle mettent en lumière le fonctionnement topique des items lexicaux. Ceux que l'on peut, parmi eux, relier en discours à des référents s'aligneront plutôt sur les précédents pour 
déterminer la signification desquels on fait entrer en considération les suites argumentatives possibles. Pour un verbe comme pleuvoir (exemple de notre cru mais inspiré par un exemple ducrotien bien connu, il fait beau, allons nous promener) on considérera donc non la présence et la densité de gouttelettes tombant verticalement des nuages sur la Terre, mais les suite discursives autorisées ou interdites, soit sans autre spécification $^{10}$

(5) Il pleut mais je sors me promener

(6) Il ne pleut pas donc je sors me promener plutôt que

(7) Il pleut donc je sors me promener

(8) Il ne pleut pas mais je sors me promener

Raccah en a très tôt fait l'application à des items lexicaux en apparence d'abord référentiels avant d'être argumentatifs, tel riche, à partir d'énoncés comme

(9) Il est riche, donc il peut acheter une belle voiture

à quoi l'on pourrait opposer « il n'est pas riche, mais il peut acheter une belle voiture » ou « il est riche, mais il ne peut pas acheter une belle voiture ».

Les topoï lexicaux sous-jacents aux enchaînements argumentatifs sont définis comme communs (à l'instar de la langue qui est une compétence partagée), généraux (contrairement aux énoncés particuliers dans lesquels on les voit à l'œuvre) et graduels, cette troisième propriété étant une spécificité de la théorie de l'argumentation dans la langue par rapport aux topoï aristotéliciens ${ }^{11}$. P et $\mathrm{Q}$ symbolisant arbitrairement des qualités graduelles, ils sont de forme

$< \pm \mathrm{X}$ est $\mathrm{P}, \pm \mathrm{X}$ est $\mathrm{Q}>$

Si l'on reprend l'exemple de Raccah, cela donne pour riche

$< \pm$ possession, \pm pouvoir $>$

ou

$< \pm X$ possède, $\pm X$ a des capacités d'action $>$.

Un problème majeur est celui du recours à l'intuition pour évaluer (conjointement) les enchaînements et les argumentations. Qu'est-ce qui prouve, comme le dit Raccah (2011 : 129), que l'énoncé

(10) Ce bébé est riche

est bizarre, alors que

(11) Ce bébé possède une grande fortune

ne le serait pas? (on peut au contraire facilement croire que « riche » et " posséder une grande fortune » convoquent le même topos élaboré à partir de la possession ${ }^{12}$ ).

Pour éviter de recourir à l'intuition en ce qui concerne la nature et la fréquence des argumentations effectuées à partir du mot démocratie (ou des dérivés: démocrate, démocratique, démocratiquement, démocratiser, démocratisation), Constantin de Chanay et Rémi-Giraud (2007) s'appuient sur un vaste corpus d'enchaînements récoltés grâce à des recherches avec guillemets sur Google pour de suites comme « démocratique mais » ou "pas démocratique mais ». On a proposé aussi de relier la notion de taxème à celle de topos lexical, ce que nous faisons en 1993 en affirmant la nécessité de relier les descriptions de langue à des attestations de discours. Nos deux extraits linguistiques indiquent indirectement une axiologisation. Manuel Valls relie ce qu'il appelle "apartheid» à des facteurs explicatifs négatifs ("peur», «chômage de masse», "déclin») et de ce fait le sémème /apartheid/ devient [négatif] ou [dysphorique] par 
afférence. Avec un résultat similaire François Hollande oppose quant à lui des parasynonymes ( $\mathrm{du}$ moins on suppose par dialogisme que ce sont des mots qui lui permettent d'éviter de prononcer le mot apartheid) à des valeurs positives et euphoriques (" promesse de la République », "vivre ensemble »), ce qui les contrarie ne pouvant être que négatif et dysphorique. Nous avons testé aussi quelques suites à la manière de Constantin de Chanay et Rémi-Giraud (2007) : ponctions discursives qui sont informatives par leur quantité, qu'il s'agisse d'enchaînements fréquents ou au contraire de séquences inattestées (d'argumentations impossibles ou du moins paradoxales).

Une question pourrait se poser, celle de la valorisation axiologique de deux membres d'un topos. À cette question Raccah apporte une réponse spécifique avec la notion de « champ topique élémentaire » qui traite à la fois les cas des mots intrinsèquement orientés, dits «euphoriques» ou «dysphoriques», et des mots qu'on peut dire axiologiquement neutres. Un champ topique élémentaire relie une catégorie à ces archi-valeurs, que sont bon, bien, etc., ou au contraire mauvais, mal, etc. Ces archi-valeurs évoquent très fortement les subjectivèmes proposés dès 1981 par Kerbrat-Orecchioni (1981, passim), ce qui fait un rapprochement supplémentaire entre la sémantique structurale et la théorie de l'argumentation dans la langue.

Ce que Raccah appelle "champ topique élémentaire», par exemple pour riche <potentialité, positif> ou <potentialité, négatif> (on le voit, il s'agit de deux champs opposés, 2011 : 135) est un élément du topos complexe < possession, $\square \pm$ potentialitl>, élément par lequel, dirions-nous, l'axiologie et l'idéologie viennent à l'argumentation. Cette idéologie varie en fonction du discours. En revanche le « champ topique complexe » (par exemple <potentialité, possession>), cristallisant en langue un topos dans un mot lexical, en l'occurrence riche, quant à lui, ne varie pas et est indépendant du discours. Pour employer une autre terminologie, la théorie des champs topiques lexicaux concilie les ressources axiologiques de la langue avec le fait que tout discours peut toujours les exploiter en convoquant des idéologies marginales ou déviantes. La question des doxas (nous empruntons ce pluriel à Rastier) est alors tout à la fois une question de genre discursif et une question dialogique. Voyons ce qu'il en est pour apartheid.

\section{Apartheid en corpus}

On peut se demander si le mot appartient bien à « la langue » et si oui sous quelle forme. La norme lexicographique est hésitante. Par exemple, le mot est tout bonnement absent du TLF (consulté en version électronique sur le site de CNRTL) mais il est présent dans le Petit Robert 2015, et déjà présent dans celui de 1986 où il figure en deux endroits, une première fois dans le volume de noms communs où il a pour définition

Ségrégation des populations de races différentes, en Afrique du Sud

et une seconde dans le volume des noms propres avec l'article suivant

Mot afrikaans désignant la politique de ségrégation raciale pratiquée en république d'Afrique du Sud, à partir de 1948 par Malan et renforcée par ses successeurs Verwoerd et Voster.

Il vérifie en effet une propriété typique couramment reconnue aux noms propres (voir Kleiber 1996), celle de renvoyer à un référent unique : nommément dans les deux cas, le régime d'Afrique du Sud; mais il vérifie aussi une propriété tout aussi typique des noms communs, il a un sens : les deux définitions comportent les traits [ségrégation] et [race]. Le mot d'apartheid, vu comme nom propre, est particulièrement peu adapté à être étudié 
aussi bien par la sémantique structurale que par la théorie de l'argumentation dans la langue. Mais vu comme nom commun, c'est l'inverse. Or il semble que le mot ait évolué dans cette direction.

\section{Voici comment le même dictionnaire définit l'apartheid en 2015 :}

Ségrégation des populations basée sur le critère de la couleur de la peau en Afrique du Sud (pratiquée officiellement jusqu'en 1990). «l'apartheid est l'expression institutionnelle de l'idéologie raciste coloniale la plus brutale » (Ziegler).

PAR EXTENSION Un apartheid linguistique, social.

Outre la date de fin officielle de la politique de ségrégation, on remarquera essentiellement trois choses. D'abord, la disparition du mot « race » (peut-être à cause de son éviction du texte de la constitution française ${ }^{13}$ ) au profit du critère à la fois moins impliquant et plus précis de la couleur de la peau: historiquement l'apartheid s'est manifesté ainsi, par des écriteaux "white only » ou des assignations «black persons ». Ensuite, une citation clairement dénonciatrice alors qu'on pouvait trouver que les définitions précédentes étaient axiologiquement neutres. La présence de cette citation peut indiquer que le sens commun du dictionnaire, c'est-à-dire la doxa, non pas une mais particulière à ce genre de discours sur la langue, a choisi son idéologie. Enfin, l'introduction d'une rubrique "par extension» signale que les usages du mot pour renvoyer à autre chose qu'au référent unique du régime d'Afrique du Sud sont devenus plus courants. Comme dans le discours de Manuel Valls apartheid est introduit par un déterminant indéfini qui présuppose que le référent n'est pas unique, qu'en outre il est qualifié par une série d'adjectifs qui en modifient l'application extensionnelle, on peut supposer qu'on a affaire à un emploi qui correspond à cette évolution.

On peut dire qu'à droite comme à gauche les controverses qui ont eu lieu à propos de l'emploi de ce mot par un premier Ministre, voire l'indignation qu'il a suscitée, se rejoignent dans le fait de saisir apartheid comme un mot particulier qui renvoie à l'Afrique du Sud. Pour Nicolas Sarkozy, il est scandaleux de comparer la République française, qu'il présente comme soucieuse au contraire d'intégrer toutes les populations, à ce "régime honni ». Pour le journal web Slate ${ }^{14}$, le même mot «ne devrait jamais être dit » parce qu'il ignore l'Histoire, au même titre que "révolution culturelle » ou " Français de souche " (pour ce dernier, imputable à François Hollande). Il est vrai qu'on ne saurait nier sans mauvaise foi que le mot d'apartheid tire une bonne part de sa puissance axiologique de la référence première au régime d'Afrique du Sud ${ }^{15}$. C'est d'ailleurs à la comparaison des deux références que procède la figure d'extension. Et c'est en ce sens sans doute que Slate parle dans les deux cas d'un « écart de langage soigneusement calibré ».

La confrontation fait deux choses : elle sélectionne dans le sens « originel » des traits que l'on retrouve dans le sens "étendu»; mais elle reporte aussi une structure qu'on peut dire « actancielle » et qui assure la répartition des qualités. Il y a d'abord un agent et un patient, comme l'implique aussi le mot de "ségrégation » (l'agent opère la ségrégation, le patient la subit); les valeurs sont réparties sur cette structure: l'action de l'agent est illégitime, le patient est une victime (c'est explicite dans les mots « la plus brutale » de la définition la plus récente, ce n'est qu'implicite dans « ségrégation »), du point de vue du dictionnaire, qui prétend refléter le point de vue de la langue; la situation peut donc provoquer l'indignation ou la révolte. Par contre ce dictionnaire ne mentionne que par la bande (" officiellement pratiquée») un trait relevé par Nicolas Sarkozy, celui d'une volonté politique à la source de l'apartheid, quand bien même les afférences neutralisent ce trait («s'est imposé»). Le débat se joue en quelque sorte entre nom propre 
(responsabilité politique en Afrique du Sud, donc en France, telle serait l'accusation) et nom commun (les faits français doivent inspirer aux partisans de l'égalité une horreur aussi grande que celle qu'ils auraient eue en Afrique du Sud). Vraisemblablement, l'évolution de la langue va lentement dans le sens du nom commun. Déjà en 1996 un numéro des Cahiers anti-spécistes s'élevait dans son titre contre « l'apartheid des espèces ». On remarquera que le trait [couleur de la peau] est négligé par rapport à celui des «types d'êtres » qui incitent plutôt à mettre des races différentes (qui existent dans le monde animal ${ }^{16}$ ) sur le même plan que les espèces; les espèces à peau non nue sont par ailleurs moins concernées par la pigmentation de l'épiderme. On remarquera aussi que la responsabilité de l'exclusion, grave dans un contexte politique (en particulier dans le contexte français où la devise de la République contient le mot «égalité »), l'est moins dans le contexte des relations entre humains et espèces autres qu'humaines, un peu comme si le domaine des pratiques culturelles et le domaine politique y étaient l'un à l'autre étanches.

Le mot apartheid appartient-il au système de la « langue française », et corrélativement, peut-on d'une part lui appliquer une analyse différentielle qui se fonderait sur ses relations avec d'autres sémèmes au sein d'un taxème de cette langue, et peut-on d'autre part l'envisager du point de vue de la théorie de l'argumentation dans "la langue " (laquelle ?) ? Les deux badges insèrent le mot dans un slogan en anglais. Historiquement parlant le mot n'est pas plus anglais que français. Selon le Petit Robert c'est un mot afrikaans, dont on apprend par ailleurs qu'il est décomposable, "heid» étant une déformation du suffixe anglais "hood» qui sert à former des abstraits et «apart» un emprunt au néerlandais, lui-même redevable à l'adverbial français «à part ». C'est donc déjà un mot congénitalement cosmopolite. Morphologiquement on peut y trouver l'idée de séparation, mais non le critère de cette séparation (race, couleur de peau - ou, dans les extensions, espèce, ethnie, résidence...). Comme souvent la motivation morphologique tourne très court. C'est un mot régi par l'arbitraire de ses morphèmes, quelle que soit la manière dont on le prononce en français ([apartèd], [apartèjd], ou comme Manuel Valls [apartajd]) : il n'entretient en effet aucune relation sémantique avec pinède, treille ou paille

Son caractère cosmopolite pose aux évolutions de la sémantique structurale le problème des mots internationaux. Apartheid aurait en fait un fonctionnement hybride. Comme nom propre, il serait international et renverrait à la réalité très singulière du régime ségrégationniste d'Afrique du Sud privilégiant les Blancs et excluant les Noirs. Comme nom commun, il s'intégrerait à une langue particulière et généraliserait sa signification. La question reste bien sûr ouverte de savoir si en corpus on doit l'interpréter plutôt comme nom commun (ce que font les défenseurs de manuel Valls) ou comme nom propre (ce que font ses détracteurs).

41 Cette question se pose aussi bien pour les vœux de Manuel Valls que pour les badges. Les modifications adjectivales (" un apartheid territorial, social, ethnique ») feraient pencher en faveur du fonctionnement comme nom commun dans cette allocution. Toutefois, ce même critère conduirait à opposer les deux badges, ce qui est contre-intuitif, d'autant plus qu'il devient courant d'employer apartheid à propos de la situation israélopalestinienne. On peut ainsi songer à un fonctionnement « dialogique ». Manuel Valls fait référence explicitement à son propre discours («ce que j'évoquais en deux mille cinq déjà : un apartheid») en s'attribuant le même mot d'apartheid pour décrire la même réalité dix ans auparavant. Il aurait pu citer Jean-Pierre Chevènement, source antérieure : 
La France s'est cassée. Socialement et spatialement. [...] La ségrégation spatiale redouble ainsi la ségrégation sociale. [...] Il faut donc se donner tous les moyens de lutter à long terme contre l'apartheid social. Et, puisqu'il revêt de plus en plus la forme d'un apartheid spatial, il faut repenser notre organisation urbaine. Relever l'idéal de la citoyenneté (26 mai 1998, cité par Genestier, Ouardi et Rennes 2007 : 73)

Ensuite il utilise dans ce contexte le mot "fracture», à distance certes de l'adjectif "social ", mais qui pour décrire une scission à l'intérieur de la société française évoque immanquablement la campagne de Jacques Chirac en 1993 autour de l'expression «facture sociale » sous l'impulsion d'Henri Guaino (expression initialement due à Marcel Gauchet). La perspective entre fracture et apartheid est néanmoins différente, l'emploi d' apartheid étant certainement un peu plus accusateur. Une fracture, c'est pathologique mais pas scandaleux : on espère la réduire (il ne faut pas se révolter mais réfléchir et soigner). Un apartheid, ça indigne : il ne se réduira pas si on ne se révolte pas (il faut se révolter).

Même si le mot d'apartheid commence à s'intégrer au français, il y reste une sorte de singleton importé et les classes de parasynonymes comme celle qu'offre François Hollande, où du reste il ne figure pas, restent discursives sans être encore stabilisées. Ce sont toutefois de bons embryons de classes où traquer des isotopies et des contrastes. Le caractère éminemment discursif et singulier des corpus dont nous disposons n'est pas un handicap, bien au contraire. On dispose en effet de trois moyens pour étudier la signification, laquelle ne peut pas s'observer directement : la confrontation du mot avec son référent en discours (c'est de première manière dont la signification en langue s'abreuve au sens en discours), les afférences contextuelles (c'est ainsi qu'on comprend toujours le mot «schtroumpf » dans la BD du même nom), le contraste avec d'autres mots $\mathrm{du}$ même taxème (qui sont rappelons-le pertinents «dans une situation de communication donnée »).

Pour ce qui est du premier point, la confrontation avec le référent pour essayer de cerner les traits dominants de la catégorie, on a deux référents avec lesquels confronter le mot. Le premier est celui d'Afrique du Sud, qui permet d'extraire les traits [illégitime] [séparation] [raciale], ce dernier trait étant remplacé par le Petit Robert par « fondé sur le critère de la couleur de la peau »; dans l'esprit de tout un chacun, cela correspond à « de couleur» vs «blanc» (le premier étant un euphémisme et le second une hyperbole). Il concerne aussi, ce qui est impliqué par [séparation] et par [racial], une [pluralité de personnes] (cette pluralité est conservée dans les extensions produites par Manuel Valls ou sur les badges, généralisée en pluralité des vivants dans l'apartheid des espèces »). On peut aussi dire que l'apartheid d'Afrique du Sud était volontaire (c'était une politique), trait neutralisé par le discours de Manuel Valls («s'est imposé ») mais pas par celui des badges.

Pour ce qui est du second point, les afférences contextuelles, on peut comme cela a été abordé plus haut dire qu'/apartheid/ adopte les traits [dysphorique] de maux, rongent, fracture, défi, tension, etc. ${ }^{17}$, et [adversité] attenant à défi, tensions qui couvent (association de mots qui évoque l'incendie imminent), émeutes; l'ensemble stigmates, relégation, ghettos et plus tard discrimination compose une isotopie de l'exclusion à laquelle s'intègre / apartheid/.

Enfin, pour le troisième point, on dispose du taxème des euphémismes d'évitement de François Hollande, du moins si l'on accepte dialogiquement que c'en sont. Ce taxème de substitution composé de "ségrégé, séparé, écarté, mis de côté» insiste sur l'idée 
d'exclusion (éviction spatiale) singulière par rapport à un groupe dont rien n'est dit ni suggéré. Au contraire apartheid suggère que les exclus sont rejetés par un groupe dominant, ce qui est sans doute plus difficile à accorder à une perspective politique socialiste, même si ce rejet n'a pas été politiquement choisi mais« imposé » à l'insu de tous.

Que nous enseignent les images? D'une part que le mot d'apartheid, on l'a compris, est transposable hors du domaine sud-africain. Et d'autre part, que le mot d'apartheid prend affectivement le parti des victimes. Dans le badge $n^{\circ} 2$ cette victime est identifiée trois fois : par le mot Palestine, par le drapeau palestinien en fond central, par les quatre couleurs noir, vert, rouge et blanc du slogan du pourtour et de son fond qui reprennent les quatre couleurs du drapeau. La victime apparaît sous une forme étatique qui confère une sorte de légitimité au poing levé de la révolte et ainsi à la révolte elle-même. L'agent opérateur (Israël) est passé sous silence. Sur le badge $n^{\circ} 1$ il est nommé par voie adjectivale ( I Israeli ») mais la Palestine n'est identifiée qu'une fois, indirectement, par le biais de la mention de Gaza (théâtre prototypique de la situation israélo-palestinienne). Gaza, synecdoque de la Palestine, apparaît isolé dans l'image, déjà par sa graphie oblique, mais surtout parce qu'il est, seul ainsi détaché, sur fond d'un cœur rouge. Ce cœur est très pictogrammatique: il est plus proche du cœur de jeux de carte que de l'organe des cardiologues ou des énoncés synonymes de «J'aime $\mathrm{X}$ » et semble spécialisé dans l'affect amoureux, ce qui fait un pendant on ne peut plus antithétique à l'affect de révolte du poing levé du badge $n^{\circ} 1$. Le résultat référentiel est le même : on se révolte aux côtés de la Palestine ${ }^{18}$, on aime Gaza. Dans ces deux images le «script actanciel » comprenant entre autres un agent et un objet n'est pas réservé aux seules définitions linguistiques. Il en va de même des axiologies. En se plaçant du côté de la victime que l'on aime ou qui se révolte, les deux images reconduisent une opposition qui traverse la langue et axiologise à l'inverse les uns des autres les mots du choix.

Soit en effet l'idée de tri, c'est-à-dire d'un critère pour retenir certains éléments soumis au tri mais pas d'autres. Elle n'est jamais neutre dans la langue. On a affaire soit à des mots positifs (" euphoriques» pour Raccah 2011) désignant le fait de retenir, soit à des mots négatifs et « dysphoriques » qui se placent comme le fait apartheid du côté du rejet. Dans les deux cas il y a une hiérarchisation, vue favorablement par les premiers et négativement par les seconds. Ce qui sélect, de choix, classe, est à la fois retenu et meilleur que le reste. À l'opposé le rebut, le déchet, ce qui est éliminé, etc., est écarté et dévalorisé.

On retrouve apparemment pour le tri la même ambivalence topique que Raccah détectait sous le mot riche pour la possession: selon les discours et donc selon les idéologies, l'exclusion est vue positivement ou négativement. Toutefois, il convient de mentionner deux choses.

La première est que les deux point de vue ne sont pas également représentés dans l'univers discursif. Comme Constantin de Chanay et Rémi-Giraud l'ont observé pour le mot démocratie (2007), qui fournit un « argument sans réplique » (on est forcément pour), de même le mot apartheid semble fournir une "dénonciation sans réplique " (on est forcément contre). Les suites apartheid mais, apartheid mais pas, les enchaînements sur pseudo-apartheid, les séquences oui à l'apartheid, etc., ne permettent pas d'observer d'argumentation pro-apartheid (comme pour démocratie on pouvait s'y attendre, mais il est toujours plaisant de le vérifier). Les topoï possibles ne sont pas tous exploités en discours, autrement dit la doxa, potentiellement plurielle, présente des zones d'homogénéisation et de cohérence topique transdiscursive. 
51 La seconde chose à mentionner est que tous les exclus ne sont pas aussi illégitimes les uns que les autres. On se rappelle la publicité Vittel «Buvez, éliminez! » : il y a des déchets qui méritent qu'on les traite comme déchets, d'autres non. Cela tient déjà au genre de discours (médical, écologique, etc.), mais pas seulement. Chaque fois que l'on parle de tri il y a au moins deux points de vue à faire entrer en ligne de compte d'une part le point de vue de celui qui opère le tri, qui garde sciemment certaines choses et en élimine d'autres ; et, d'autre part, le point de vue de celui qui énonce le tri, qui peut théoriquement en être partisan ou non. De l'un à l'autre s'étend un spectre d'arguments tout faits. Il semblerait que ces deux points de vue puissent se dissocier lorsque l'on est au milieu : les mots alors peuvent y argumenter ou y contre-argumenter et les corpus permettront d'observer toutes les cartes topiques. C'est le cas d'un mot comme sélection, qu'on trouve selon les idéologies en bonne ou en mauvaise part. Mais aux deux extrêmes de la chaîne, les points de vue semblent se spécifier et coaguler. D'un côté, des lexies comme de choix, qui disent uniment la légitimité du tri et le point de vue favorable de celui qui l'énonce. De l'autre, des mots comme apartheid qui disent à la fois l'illégitimité du choix et la défaveur dans laquelle celui qui parle tient ce choix. À l'évidence, il y a là des raisons de cohérence discursive qui interdisent d'approuver ce que l'on présente comme illégitime et inversement. Mais ce n'est pas le seul facteur. Il suffit de se rappeler que pour les discours national-socialistes les Juifs étaient qualifiés de "rats», de "rebuts", de «lie de l'humanité » pour se dire que ce fond discursif peut légitimer l'idée de solution finale ou tout autre représentant discursif (comme peut l'être dans un autre contexte le mot de ratonnade, qui malgré les apparences n'est pas dysphorique pour tout le monde). Ce qui en la matière semble compter avant tout est que l'espace discursif, où se récoltent les corpus, est un espace démocratique dans lequel ne se remet pas en cause l'égalité des humains ${ }^{19}$. C'est une sorte de "doxa de fond» dont seraient révélateurs les champs topiques élémentaires, tandis que les topoï révéleraient les idéologies endossables au sein de cette doxa.

\section{Conclusion}

52 L'exemple du mot apartheid montre une voie pour accorder la théorie de l'argumentation dans la langue et une sémantique différentielle exercée sur un petit corpus de discours oraux et d'images. La notion de champ topique élémentaire, tout comme les subjectivèmes proposés par Kerbrat-Orecchioni, fait le lien entre les deux démarches.

53 La théorie de l'argumentation dans la langue sous cette double forme est une formalisation théorique de ce qui reste une étude empirique où l'on doit corréler hypothèses et observations. À la lumière d'un petit corpus argumentatif - deux allocutions, deux badges militants - nous avons formulé des hypothèses topiques sur les mots du tri, soit un spectre s'étendant d'un champ topique favorable (pour de choix) à son inverse (pour apartheid) avec des mots hésitant les deux (du plus neutre au plus favorable : sélection, sélectif, sélect) autorisant des idéologies opposées contrastant par leur topoï favorables ou défavorables à la hiérarchisation et à l'exclusion.

54 Il apparaît quand même que la langue cristallise un espace de parole idéologiquement conditionné où certains discours sont irrecevables (pro-apartheid comme antidémocratique), ce qui revient à dire que l'idéologie se reflétant in fine dans la langue relève avant tout de pratiques, discursives comme extra-discursives. Ces pratiques sont manifestement relatives à des ensembles sociaux dont il est permis de penser qu'ils sont 
les sources des axiologies cristallisées par le système de la langue. La portée politique des discours autorisée par l'argumentation est peut-être, tout à la fois en nommant et en dénonçant (par exemple en employant le mot apartheid), de chercher à infléchir par les pratiques discursives, dont on espère qu'elles vont mobiliser et rallier, le fait accompli des pratiques extra-discursives qu'ils font apparaitre.

\section{BIBLIOGRAPHIE}

Anscombre Jean-Claude et Ducrot Oswald, L'argumentation dans la langue, Bruxelles, P. Mardaga, 1983.

Benveniste Émile, Problèmes de linguistique générale, tomes I et II, Paris, Gallimard, 1966 et 1974. Bruxelles Sylvie et Constantin de Chanay Hugues, « Averca de la teoría de los topoi : estado de la cuestión » [sur la théorie des topoï : état des lieux des lieux, Escritos n ${ }^{\text {os }} 17-18$, décembre 1998, Universidad Autónoma de Puebla, Mexico. pp. 349-383.

Constantin de Chanay Hugues, "Sens lexical et argumentation : des CNS aux topoi », in Christian Plantin (éd.), Lieux communs, Stéréotypes, Topoi, Kimé, 1993, pp. 290-301.

Ducrot Oswald, « Opérateurs argumentatifs et analyse de textes », in Current Issues in Linguistic Theory, 103, Amsterdam/Philadelphia, John Benjamins, 1993, pp. 45-63.

Genestrier Philippe, Ouardi Samira et Rennes Juliette, « Le paradigme localiste au secours de l'action publique démocratique », Mots $\mathrm{n}^{\circ}$ 83, 2007, pp. 69-80.

Constantin de Chanay Hugues et Rémi-Giraud Sylvianne, « Démocratie et ses dérivés. De la dénomination à l'argument sans réplique ?», Mots. Les langages du politique, 2007, nº 83, pp. 81-99.

Kerbrat-Orecchioni Catherine, De la sémantique lexicale à la sémantique de l'énonciation, Thèse de Doctorat d'État, Lille, 1977.

Kerbrat-Orecchioni Catherine, L'énonciation. De la subjectivité dans le langage, Paris, Armand Colin, 1981.

Kleiber Georges, La sémantique du prototype, Paris, PUF, 1990.

Kleiber Georges, « Noms propres et noms communs : un problème de dénomination », Meta : journal des traducteurs / Meta: Translators' Journal, vol. 41, $\mathrm{n}^{\circ}$ 4, 1996, pp. 567-589.

Morel Mary-Annick et Danon-Boileau Laurent, Grammaire de l'intonation, Paris, Ophrys, 1998.

Perelman Chaïm et Olbrechts-Tyteca Lucie, Traité de l'argumentation, Éditions de l'université de Bruxelles, 1988.

Raccah Pierre-Yves, « Signification, sens et connaissance : une approche topique », Cahiers de Linguistique Française n 11, 1990, pp. 179-198.

Raccah Pierre-Yves, « Racines lexicales de l'argumentation : la cristallisation des points de vue dans les mots », Verbum, revue de linguistique, 2011, 1 n 32, pp. 119-141.

Rastier François, Sémantique interprétative, Paris, PUF, 1987. 
Rastier François, La Mesure et le grain. Sémantique de corpus, Paris, Champion, 2011.

Saussure Ferdinand de, Cours de linguistique générale, Paris, Payot, [1916] 1972.

\section{NOTES}

1. Notre transcription, ponctuation ajoutée.

2. https://www.youtube.com/watch?v=QIrS49qJIU4. Nous utilisons les conventions de transcription Icor (http://icar.univ-lyon2.fr/projets/corinte/ documents/2013_Conv_ICOR_250313.pdf).

3. Prononcé [apartajd].

4. $h t t p s: / / w w w . y o u t u b e . c o m / w a t c h ? v=\_f R E N L z V E n c$. On en trouve un compte rendu sur le site web du Monde (http://www.lemonde.fr/politique/article/2015/02/05/hollande-annonce-uneinitiative-franco-allemande-sur-l-ukraine_4570054_823448.html).

5. Photographies de l'auteur de badges courants achetés sur le site Zazzle.com en mars 2015.

6. Nous choisissons ici de placer les sémèmes entre barres obliques à titre d'unités de langue (comme les transcriptions phonologiques par opposition aux transcriptions phonétiques), et les sèmes entre crochets à la fois pour ne pas préjuger de leur statut formel et par analogie avec les traits distinctifs de la phonologie, eux aussi entre crochets.

7. Nous évitions à dessein de parler de «domaine » dont la sémantique interprétative a donné une définition technique.

8. Indépendamment des habitudes alimentaires générales combinant dans les plats principaux des produits animaux et des produits végétaux, il existe un « couscous de la mer ».

9. Ou animale si on considère que les quenelles les plus typiques dont les quenelles de brochet.

10. C'est-à-dire sans supposer qu'on va ramasser des escargots ou qu'on désire être mouillé.

11. Sur la gradualité, voir en particulier Raccah (1990 passim).

12. Et pour ne rien résoudre les seuls exemples que Google donne pour «ce bébé est riche » sont des exemples méta issus de l'article de Raccah.

13. On craignait que la présence du mot «race » ou de l'adjectif « racial» n'y présupposent l'existence des races.

14. http://www.slate.fr/story/98423/apartheid-francais-de-souche-revolution-culturelle

15. Il est juste de dire toutefois que la référence historique peut jouer aussi dans un autre sens : le mot apartheid figure dans le programme de la Maison des métallos (théâtre parisien) pour dénoncer, comme sur nos badges, les relations israélo-palestiniennes, non seulement parce que cela serait une ségrégation comparable, mais aussi "parce que c'est une histoire qui finit bien » (par l'abolition; commentaire de Thomas Kopp, directeur de la communication de la Maison des métallos).

16. Précisément extra-humain.

17. Nous ne cherchons pas à être exhaustif. Il n'est pas sûr d'ailleurs que nous devions l'être : l'oral n'a pas les mêmes conditions de production et de réception qu'un texte écrit (et a fortiori qu'un poème), même si cette intervention-là était prévue pour la presse, donc pour être retranscrite et probablement résumée (d'où l'utilité d'y semer des points saillants, apartheid en est un), et s'il est difficile d'ignorer que tout se retrouvera tôt ou tard en vidéo sur les réseaux sociaux.

18. Le présence du mot «free » opposé à « apartheid » exclut que l'on fasse un contresens sur la cible du poing en pensant que ce poing se lève contre la Palestine.

19. Il n'en a pas toujours été ainsi. En 1931, le sujet de philosophie donné à Lille au baccalauréat était libellé ainsi : "Y a-t-il des races supérieures et des races inférieures? Si oui, quel est le devoir des premières envers les secondes?». 


\section{RÉSUMÉS}

Cet article s'intéresse au mot apartheid tel qu'il apparaît dans différents discours - une allocution en français par Manuel Valls et deux slogans anglais figurant sur des badges - qu'il entend étudier à la fois dans une perspective sémantique structurale et dans une perspective argumentative, selon les approches différentielles du niveau sémantique des langues et l'approche argumentative de la signification défendue par Pierre-Yves Raccah. Nous interrogeons ainsi d'un côté les relations entre information et argumentation, et de l'autre les relations entre axiologie et signification à travers un exemple de polysémioticité des topoi, ainsi que l'a suggéré François Rastier.

In this paper we consider the word apartheid which occurs in different discourses - one French allocution of Manuel Valls and two English slogans on badges - and we intend to study it both in a structural semantic and in an argumentative perspective, according respectively to differential approaches of semantic level in languages and with the argumentative approach of signification defended by Pierre-Yves Raccah. We question thus the relations between the axiology and the signification through an example of the polysemioticity of topoi, as suggested by François Rastier.

\section{INDEX}

Mots-clés : Discours, signification lexicale, argumentation, topoi

Keywords : Discourses, lexical signification, argumentation, topoi

\section{AUTEUR}

HUGUES DE CONSTANTIN DE CHANAY

Université de Lyon 2 